\title{
ANALYSIS OF THE QUALITY OF CHEST COMPRESSIONS DURING RESUSCITATION IN AN UNDERSTAFFED TEAM — RANDOMISED CROSSOVER MANIKIN STUDY
}

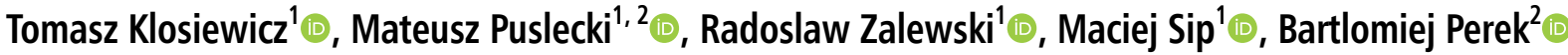 \\ ${ }^{1}$ Department of Medical Rescue, Poznan University of Medical Sciences, Poland \\ ${ }^{2}$ Department of Cardiac Surgery and Transplantology, Poznan University of Medical Sciences, Poland
}

\begin{abstract}
INTRODUCTION: According to the chain of survival, chest compressions (CCS) are crucial in every cardiac arrest patient. It is very challenging to provide high-quality resuscitation in a two-paramedic team. The task of an automatic chest compression device (ACCD) is to relieve the rescuer and improve the quality of CCs. Its influence on the quality of the whole resuscitation as well as the survival of patients is still subject to discussion worldwide. This study aimed to assess the quality of CCs during resuscitation in a two-paramedic team using ACCD.

MATERIAL AND METHODS: This research was designed as a prospective, randomised, cross-over, high-fidelity simulation study. Fifty-two double paramedic teams took part in the research. The role of the participants was to conduct full advanced resuscitation in a human patient's simulator. Each team provided resuscitation twice. Once with an ACCD and once using manual compressions. Chest compression quality parameters, as well as chest compression fraction (CCF), were measured.
\end{abstract}

RESULTS: Statistically significant differences were found between manual and automated compressions in: mean depth (48 $\pm 4 \mathrm{~mm}$ vs. $56 \pm 3 \mathrm{~mm}, \mathrm{p}<0.0001)$, mean rate $(117 \pm 9 \mathrm{~mm}$ vs. $103 \pm 1 \mathrm{~mm}, \mathrm{p}<0.0001)$, percentage of CC with correct depth $(46 \pm 25$ vs. $87 \pm 13, p<0.0001)$, rate $(72 \pm 22$ vs. $96 \pm 4$, $p<0.0001)$, and recoil $(55 \pm 23$ vs. $89 \pm 13, p<0.0001)$. CCF was also higher when the ACCD was used (74 $\pm 7 \%$ vs. $83 \pm 2 \%, p<0.0001)$.

CONCLUSIONS: The use of an ACCD increases the quality of compressions by improving CCF, chest recoil, and the percentage of compressions performed with adherence to guidelines.

KEY WORDS: cardiopulmonary resuscitation; automated chest compression device; paramedic; quality assessment; simulation study

Disaster Emerg Med J 2020; 5(1): 24-29

\section{INTRODUCTION}

Sudden cardiac death $(\mathrm{SCD})$ remains the major public health burden worldwide [1]. SCD accounts for $50 \%$ of all cardiovascular mortality. It is the first presentation of cardiac disease in $25-50 \%$ of cases and the most common outcome of sudden cardiac arrest (SCA). Despite the development of technol- ogy and advances in knowledge, survival remains worryingly low: it has been estimated at $6-10 \%$ for out-of-hospital sudden cardiac arrest (OHCA) and up to $24 \%$ for in-hospital arrest (IHCA) [2].

According to the chain of survival, four crucial links must be made to improve the outcome. These are: (1) early recognition and activation of emer- 
gency medical services, (2) high-quality chest compressions (CCs), (3) rapid defibrillation, and (4) good quality post-resuscitation care.

More effective resuscitation strategies need to be found. A mass media campaign targeting the community's awareness of heart attack symptoms translates into a reduction in the incidence of OHCA [3]. In countries where educating school children in cardiopulmonary resuscitation (CPR) is mandatory, lay resuscitation is done in $60-75 \%$ of cases [4]. In places where public access to defibrillation programs have been successfully implemented, survival has increased almost threefold [5].

Some authors suggest that the quality of CC is not appropriate, even with implementation of feedback devices. Especially in a team where only two paramedics provide resuscitation in prehospital conditions $[6,7]$. The implementation of CPR techniques that entirely follow the European Resuscitation Council (ERC) Guidelines in two-paramedic teams is challenging due to the limited human resources.

The task of an automatic chest compressions device (ACCD) is to relieve the rescuer and improve the quality of chest compressions. Their influence on the quality of the whole CPR as well as the survival of patients is still subject to discussion worldwide.

This study aims to assess the quality of chest compressions during resuscitation in a two-paramedic team using an ACCD.

\section{Institutional statement \\ MATERIAL AND METHODS}

The study protocol was approved by the Institutional Review Board of Poznan University of Medical Sciences (no. KB764/19).

\section{Study design}

This research was designed as a prospective, randomised, cross-over, high-fidelity simulation study. The Resusci Anne Advanced Skill Trainer (Laerdal Medical AS, Stavanger, Norway) human simulator was used during the study. The manikin generates a pulse on the carotid artery, respiration, and heart rhythm, including ventricular fibrillation and sinus rhythm. Airway management with supraglottic devices and upper limb adapted to insert an intravenous catheter were prepared. During all scenarios, the simulator was placed on a flat floor in a welllit room.

\section{Study protocol}

Fifty-two double teams were invited to take part in the study. The included participants were paramedics working in two-member ambulance teams, with at least three years of work experience.

The 10-minute adult male cardiac arrest scenario was created. Pulseless electrical activity was the initial rhythm, and it converted automatically to ventricular fibrillation in the fifth minute of the scenario. After 10 minutes the scenario finished, regardless of the participants' actions.

In the pre-briefing the teams were instructed on how to use the equipment, and participants could practice each of the elements on the human simulator. Printed educational materials of the Advanced Life Support (ALS) algorithm according to the 2015 ERC Guidelines were provided. The priorities of the proceedings were indicated, and the expectations were presented to the paramedics.

Each team took part in the same scenario two times. The first time, paramedics provided manual CCs (experimental group), and for the second they used the automated manual chest compression device (LUCAS 2 Chest Compression System, Physio-Control, Redmond, Washington, USA) - control group. The order of the scenarios was decided by means of the team leaders chooosing one of two sealed envelopes in which there were cards with the words "First LUCAS" or "First without LUCAS". Between the scenarios, the teams rested for at least 20 minutes.

\section{Measurements}

During the test, the following parameters were monitored with Session Viewer Software 6.2.6400 (SimVentures 2019): chest compression fraction (CCF) (1), correct hand position (3), mean number (4), depth (5), rate (6) of compressions, percentage of compressions at correct depth (7), recoil (8), and at correct rate (9).

During the scenario the time was measured using a stopwatch. The stopwatch was switched on at the moment when the researcher said the word "Start" (time 0:00).

Each scenario was video recorded (Samsung Galaxy A5, Samsung, South Korea) and then downloaded to a laptop computer. Video clips were reviewed using Windows Movie Maker (Microsoft, CA, USA). This programme permits real time playback and a frame-by-frame review of each test. This allowed precise measurement of the steps in the scenario. 


\begin{tabular}{|c|c|c|c|}
\hline Variable & $\begin{array}{c}\text { Manual } \\
\text { CC }\end{array}$ & $\begin{array}{l}\text { Automated } \\
\text { CC }\end{array}$ & $P$ value \\
\hline CCF [\%] & $74 \pm 4$ & $83 \pm 2$ & $<0.0001$ \\
\hline $\begin{array}{l}\text { Number of } \\
\text { compressions }\end{array}$ & 45008 & 37238 & $<0.0001$ \\
\hline Mean depth & $48 \pm 4$ & $56 \pm 3$ & $<0.0001$ \\
\hline $\begin{array}{l}\% \text { of CC with correct } \\
\text { depth }\end{array}$ & $46 \pm 25$ & $87 \pm 13$ & $<0.0001$ \\
\hline Mean rate & $117 \pm 9$ & $103 \pm 1$ & $<0.0001$ \\
\hline $\begin{array}{l}\% \text { of CC at correct } \\
\text { rate }\end{array}$ & $72 \pm 22$ & $96 \pm 4$ & $<0.0001$ \\
\hline $\begin{array}{l}\% \text { of CC complete } \\
\text { recoiled }\end{array}$ & $55 \pm 23$ & $89 \pm 13$ & $<0.0001$ \\
\hline
\end{tabular}

CC — chest compression; CCF — chest compression fraction

\section{Statistical analysis}

First, the quantitative variables were checked for normality with the use of the Shapiro-Wilk W test. If they satisfied normal distribution criteria, they were expressed as means \pm standard deviation. The categorical variables were expressed as the numbers (n) with percentages (\%). For statistical analysis Student's t-test was used as appropriate. A P value less than 0.05 was considered as significant. The analysis was performed using Statistica 12 software (Tibco Inc., Tulsa, OK, USA).

\section{RESULTS}

A total of 52 trials were conducted in each group. In the control group eight attempts were rejected due to a measurement error. The remaining 46 tests were eligible for further analysis. In the experimental group the total number of compressions was 82.246. The mean number of compressions performed during one scenario was $864 \pm 23.44$ for the control group and $866 \pm 72.40$ for the experimental group. A detailed distribution of the results is presented in Table 1 . In the group in which manual CC were provided, all CC quality parameters as well as CCF were lower than in the group in which ACCD was used.

\section{DISCUSSION}

To the best of the authors' knowledge, this was the first randomised crossover trial to indicate the difference in quality of CCs during resuscitation in understaffed ambulance teams with and without ACCD.
High-quality CC increases the chances of survival by 1.5-4 times [8]. Few determinants of the quality have been defined based on improved clinical outcome. These are: CC rate, depth, full chest recoil, and minimising interruptions. The ERC recommends that providers should perform compressions with a rate between 100-120/min and depth of 5-6 cm.

The aim of correct CC is to maintain the perfusion of brain tissue and proper coronary perfusion pressure (CPP). The CPP is defined as the difference between pressure in the aorta (origin of the coronary arteries) and in the right atrium (where coronary venous blood returns) [9]. The CPP value that is necessary to obtain return of spontaneous circulation (ROSC) was determined at $15 \mathrm{~mm} \mathrm{Hg}$ [10]. Only that parameter allows increased survival among patients with SCA. Ensuring high-quality CC requires a good team assembly and minimisation of interruptions. It is reasonable to use CPR feedback or prompt devices to achieve good quality. Some authors, however, suggest that it should only be considered as part of a broader system of CPR improvements [11].

The results of an in-hospital patient study showed that the mean depth of compression deteriorated between 1.5 and 3 min after starting CPR. For that reason the ERC recommends that chest compressors should switch every two minutes to avoid fatigue [12].

Neither ERC nor American Heart Association (AHA) recommend routine use of ACCD $[13,14]$. They suggest that it might be helpful in cases in which poor quality of CC is suspected. These are: prolonged CPR (hypothermia, intoxications), CPR during transportation, simultaneously performed advanced procedures like percutaneous coronary interventions or thrombolysis, and limited space.

In the study by Gassler et al. it was shown that among three ACCDs only the LUCAS 2 fulfilled all ERC recommendations with fraction of correct compressions at $21 \pm 15 \%$ [15]. The guidelines in no way refer to a reduced number of members in the resuscitation team as a condition in which it is difficult to provide CPR.

In this study, in the experimental group mean depth of compressions was lower - just above the lower range compared to the control group. Talikowska et al. found that, even if rescuers provided compressions within the normal range, survivors demonstrated a lower mean compression rate than non-survivors [16]. 
In the study performer by Idris et al., mean chest compression depth deteriorated in more than $80 \%$ of patients when the compression rate was faster than $140 / \min [17]$.

In the control group, a tendency of the paramedics to provide compressions that were too shallow was found. Similar results were also observed in the study by Mejer et al. In their research, the physicians were able to achieve a median depth of CC at the level of $44 \mathrm{~mm}$ [18].

The chest should be fully recoiled. Incomplete recoil during the decompression phase decreases mean arterial pressure, CPP, and cerebral perfusion pressures [19]. Leaving a residual pressure on the chest due to leaning results in increased intrathoracic pressure. This provokes reduced afterload. Fried et al. found that leaning was observed in $91 \%$ of the resuscitations, underscoring its all-too-common occurrence [20]. In the results presented in this paper, CC performed with ACCD was connected with significantly better chest recoil.

Chest compression fraction is the percentage of time when chest compressions were performed during resuscitation. At the moment, ERC recommends that CCF should be at least 60\% [11]. However, it should not be treated as an isolated predictor of survival. Its value should be interpreted in the context of other resuscitation activities [21]. Vaillancourt et al. found that the adjusted odds ratios of ROSC for various CCF categories were: $0-40 \%$ (reference group); $41-60 \%(1.14 ; 0.72,1.81) ; 61-80 \%(1.42$; $0.92,2.20)$; and $81-100 \%(1.48 ; 0.94,2.32)[22]$. The results of studies in different groups of patients show that higher CCF is associated with higher survival and better neurological outcome, especially in patients whose resuscitation lasts longer than 15-20 minutes [23]. In many countries prehospital emergency care is based on two paramedics or other provider teams. For those teams it seems to be impossible to maintain good quality of compressions even when the compressor switches every two minutes.

Hands-on defibrillation can increase CCF by shortening the no-flow time associated with charging the defibrillator and making a shock. Currently, more and more research is being done, which indicates that this method of defibrillation is safer than previously thought [24]. Some authors suggest that the use of Class 1 electrical insulating gloves during CPR allows safe hands-on shock if the rescuer touches the chest with only one palm
[25]. Others advise that CCF may be elongated when "charge and check" method is used during CPR [26].

Eftestol et al. reported a $50 \%$ relative reduction in the probability of ROSC in humans in cardiac arrest due to ventricular fibrillation when defibrillation was delayed for more than five seconds after cessation of chest compressions [27].

According to Bonnes et al., high-quality randomised evidence does not support a routine strategy of ACCD to improve survival or neurological outcome [28]. On the other hand, the results of a meta-analysis including almost 30,000 individuals published in 2018 show that manual compression is more effective than AutoPulse and is comparable to LUCAS in improving survival at 30 days or hospital discharge and neurological recovery. Manual compression also had a lower risk of complications like pneumothorax or haematoma formation compared to AutoPulse. ACC provided by the LUCAS device improved the quality by reducing the no-flow fraction and by improving the quality of CC [29].

The size of the resuscitation team is becoming a key factor affecting the quality of CPR. Up to now, the exact number of members has not been defined.

Many papers have analysed this problem. On their basis, it can be stated that three people in the team seem to provide the best quality. Some authors suggest that after a two-minute loop a rescuer needs six minutes of rest to resume good CC, and the team should consist of four people [30, 31].

However, the presence of three or more paramedics at the scene of OHCA was not associated with improved survival to hospital discharge when compared to crews with two paramedics [32]. Additional research is needed to determine the potential cause of this finding.

In simulation studies provided by Jo et al. it has been shown that an improvement in the quality of CC is achieved if rescuers change more frequently [33]. Chung suggests that, regardless of muscle strength, the switch should be made every minute [34]. Regular training is important because after just six months of training, knowledge and skills deteriorate [35].

\section{CONCLUSIONS}

Based on the results of the research, it can be concluded that the quality of CC in a two-person resuscitation team is insufficient and the use of ACCD during CPR increases the quality of CC by improving 
CCF, chest recoil, and percentage of compressions performed with adherence to guidelines.

\section{REFERENCES}

1. Wong CX, Brown A, Lau DH, et al. Epidemiology of Sudden Cardiac Death: Global and Regional Perspectives. Heart Lung Circ. 2019; 28(1): 6-14, doi: 10.1016/j.hlc.2018.08.026, indexed in Pubmed: 30482683.

2. Al-Khatib SM, Stevenson WG, Ackerman MJ, et al. 2017 AHA/ACC/HRS guideline for management of patients with ventricular arrhythmias and the prevention of sudden cardiac death: Executive summary: A Report of the American College of Cardiology/American Heart Association Task Force on Clinical Practice Guidelines and the Heart Rhythm Society. Heart Rhythm. 2018; 15(10): e190-e252, doi: 10.1016/j. hrthm.2017.10.035, indexed in Pubmed: 29097320.

3. Benjamin EJ, Blaha MJ, Chiuve SE, et al. American Heart Association Statistics Committee and Stroke Statistics Subcommittee. Heart Disease and Stroke Statistics-2017 Update: A Report From the American Heart Association. Circulation. 2017; 135(10): e146-e603, doi: 10.1161/ CIR.0000000000000485, indexed in Pubmed: 28122885.

4. Wissenberg M, Lippert FK, Folke F, et al. Association of national initiatives to improve cardiac arrest management with rates of bystander intervention and patient survival after out-of-hospital cardiac arrest. JAMA. 2013; 310(13): 1377-1384, doi: 10.1001/jama.2013.278483, indexed in Pubmed: 24084923.

5. Kishimori T, Kiguchi T, Kiyohara K, et al. Public-access automated external defibrillator pad application and favorable neurological outcome after out-of-hospital cardiac arrest in public locations: A prospective population-based propensity score-matched study. Int J Cardiol. 2020; 299: 140-146, doi: 10.1016/j.ijcard.2019.07.061, indexed in Pubmed: 31400888.

6. Cheng A, Hunt EA, Grant $D$, et al. International Network for Simulation-based Pediatric Innovation, Research, and Education CPR Investigators. Variability in quality of chest compressions provided during simulated cardiac arrest across nine pediatric institutions. Resuscitation. 2015; 97: 13-19, doi: 10.1016/j.resuscitation.2015.08.024, indexed in Pubmed: 26417701.

7. Dabrowski M, Sip M, Dabrowska A, et al. It is impossible to follow the ERC algorithm in a two-paramedics ambulance team. Resuscitation. 2017; 118: e43, doi: 10.1016/j.resuscitation.2017.08.108.

8. Hasselqvist-Ax I, Riva G, Herlitz J, et al. Early cardiopulmonary resuscitation in out-of-hospital cardiac arrest. N Engl J Med. 2015; 372(24): 2307-2315, doi: 10.1056/NEJMoa1405796, indexed in Pubmed: 26061835.

9. Paradis NA, Martin GB, Rivers EP, et al. Coronary perfusion pressure and the return of spontaneous circulation in human cardiopulmonary resuscitation. JAMA. 1990; 263(8): 1106-1113, indexed in Pubmed: 2386557.

10. Sutton RM, Friess SH, Maltese MR, et al. Hemodynamic-directed cardiopulmonary resuscitation during in-hospital cardiac arrest. Resuscitation. 2014; 85(8): 983-986, doi: 10.1016/j.resuscitation.2014.04.015, indexed in Pubmed: 24783998.
11. Perkins GD, Handley AJ, Koster RW, et al. Adult basic life support and automated external defibrillation section Collaborators. European Resuscitation Council Guidelines for Resuscitation 2015: Section 2. Adult basic life support and automated external defibrillation. Resuscitation. 2015; 95: 81-99, doi: 10.1016/j.resuscitation.2015.07.015, indexed in Pubmed: 26477420.

12. Sugerman NT, Edelson DP, Leary $M$, et al. Rescuer fatigue during actual in-hospital cardiopulmonary resuscitation with audiovisual feedback: a prospective multicenter study. Resuscitation. 2009; 80(9): 981-984, doi: 10.1016/j.resuscitation.2009.06.002, indexed in Pubmed: 19581036.

13. Soar J, Nolan JP, Böttiger BW, et al. Adult advanced life support section Collaborators. European Resuscitation Council Guidelines for Resuscitation 2015: Section 3. Adult advanced life support. Resuscitation. 2015; 95: 100-147, doi: 10.1016/j.resuscitation.2015.07.016, indexed in Pubmed: 26477701.

14. Brooks SC, Anderson ML, Bruder E, et al. Part 6: Alternative Techniques and Ancillary Devices for Cardiopulmonary Resuscitation: 2015 American Heart Association Guidelines Update for Cardiopulmonary Resuscitation and Emergency Cardiovascular Care. Circulation. 2015; 132(18 Suppl 2): S436-S443, doi: 10.1161/CIR.0000000000000260, indexed in Pubmed: 26472994.

15. Gässler H, Kümmerle S, Ventzke MM, et al. Mechanical chest compression: an alternative in helicopter emergency medical services? Intern Emerg Med. 2015; 10(6): 715-720, doi: 10.1007/s11739-015-1238-0, indexed in Pubmed: 25918109.

16. Talikowska M, Tohira H, Finn J. Cardiopulmonary resuscitation quality and patient survival outcome in cardiac arrest: A systematic review and meta-analysis. Resuscitation. 2015; 96: 66-77, doi: 10.1016/j. resuscitation.2015.07.036, indexed in Pubmed: 26247143.

17. Idris AH, Guffey D, Aufderheide TP, et al. Resuscitation Outcomes Consortium (ROC) Investigators. Relationship between chest compression rates and outcomes from cardiac arrest. Circulation. 2012; 125(24): 3004-3012, doi: 10.1161/CIRCULATIONAHA.111.059535, indexed in Pubmed: 22623717.

18. Majer J, Smereka J, Ladny JR, et al. Quality of chest compressions during cardiopulmonary resuscitation performed by physicians: do we need to use mechanical chest compression devices? A multicenter, randomized, crossover study. Post N Med. 2018; 31(6): 314-321, doi: 10.25121/PNM.2018.31.6.314.

19. Yannopoulos D, McKnite S, Aufderheide TP, et al. Effects of incomplete chest wall decompression during cardiopulmonary resuscitation on coronary and cerebral perfusion pressures in a porcine model of cardiac arrest. Resuscitation. 2005; 64(3): 363-372, doi: 10.1016/j. resuscitation.2004.10.009, indexed in Pubmed: 15733767.

20. Fried DA, Leary M, Smith DA, et al. The prevalence of chest compression leaning during in-hospital cardiopulmonary resuscitation. Resuscitation. 2011; 82(8): 1019-1024, doi: 10.1016/j.resuscitation.2011.02.032, indexed in Pubmed: 21482010.

21. Wik $L$, Olsen JA, Persse $D$, et al. Why do some studies find that CPR fraction is not a predictor of survival? Resuscitation. 2016; 104: 
59-62, doi: 10.1016/j.resuscitation.2016.04.013, indexed in Pubmed: 27155547.

22. Vaillancourt C, Everson-Stewart S, Christenson J, et al. Resuscitation Outcomes Consortium Investigators. The impact of increased chest compression fraction on return of spontaneous circulation for out-of-hospital cardiac arrest patients not in ventricular fibrillation. Resuscitation. 2011; 82(12): 1501-1507, doi: 10.1016/j.resuscitation.2011.07.011, indexed in Pubmed: 21763252.

23. Rea T, Olsufka M, Yin L, et al. The relationship between chest compression fraction and outcome from ventricular fibrillation arrests in prolonged resuscitations. Resuscitation. 2014; 85(7): 879-884, doi: 10.1016/j.resuscitation.2014.02.026, indexed in Pubmed: 24607869.

24. Brady W, Berlat JA. Hands-on defibrillation during active chest compressions: eliminating another interruption. Am J Emerg Med. 2016; 34(11): 2172-2176, doi: 10.1016/j.ajem.2016.08.017, indexed in Pubmed: 27645811.

25. Deakin CD, Thomsen JE, Lofgren Bo, et al. Achieving safe hands-on defibrillation using electrical safety gloves--a clinical evaluation. Resuscitation. 2015; 90: 163-167, doi: 10.1016/j.resuscitation.2014.12.028, indexed in Pubmed: 25725295.

26. Dabrowski M, Klosiewicz T, Sip M, et al. The final battle. What more can we do to be victorious with cardiac arrest? Preliminary data. Anestezjologia i Ratownictwo. 2018; 12: 111-116.

27. Eftestøl T, Sunde K, Steen PA. Effects of interrupting precordial compressions on the calculated probability of defibrillation success during out-of-hospital cardiac arrest. Circulation. 2002; 105(19): 2270-2273, doi: 10.1161/01.cir.0000016362.42586.fe, indexed in Pubmed: 12010909.

28. Bonnes JL, Brouwer MA, Navarese EP, et al. Manual Cardiopulmonary Resuscitation Versus CPR Including a Mechanical Chest Compression Device in Out-of-Hospital Cardiac Arrest: A Comprehensive Meta-analysis From Randomized and Observational Studies. Ann Emerg Med.
2016; 67(3): 349-360.e3, doi: 10.1016/j.annemergmed.2015.09.023, indexed in Pubmed: 26607332.

29. Tranberg T, Lassen JF, Kaltoft AK, et al. Quality of cardiopulmonary resuscitation in out-of-hospital cardiac arrest before and after introduction of a mechanical chest compression device, LUCAS-2; a prospective, observational study. Scand J Trauma Resusc Emerg Med. 2015; 23: 37, doi: 10.1186/s13049-015-0114-2, indexed in Pubmed: 25898992.

30. Yamanaka S, Huh JiY, Nishiyama K, et al. The optimal number of personnel for good quality of chest compressions: A prospective randomized parallel manikin trial. PLoS One. 2017; 12(12): e0189412, doi: 10.1371/journal.pone.0189412, indexed in Pubmed: 29267300.

31. Hunziker S, O'Connell KJ, Ranniger C, et al. Effects of designated leadership and team-size on cardiopulmonary resuscitation: The Basel-Washington SIMulation (BaWaSim) trial. J Crit Care. 2018; 48: 72-77, doi: 10.1016/j.jcrc.2018.08.001, indexed in Pubmed: 30172964.

32. Eschmann NM, Pirrallo RG, Aufderheide TP, et al. The association between emergency medical services staffing patterns and out-of-hospital cardiac arrest survival. Prehosp Emerg Care. 2010; 14(1): 71-77, doi: 10.3109/10903120903349820, indexed in Pubmed: 19947870.

33. Jo CH, Cho GC, Ahn JH, et al. Rescuer-limited cardiopulmonary resuscitation as an alternative to 2-min switched CPR in the setting of inhospital cardiac arrest: a randomised cross-over study. Emerg Med J. 2015; 32(7): 539-543, doi: 10.1136/emermed-2013-203477, indexed in Pubmed: 25092797.

34. Kim H, You J, Chung S. Influence of rescuer strength and shift cycle time on chest compression quality. Signa Vitae - A Journal In Intensive Care And Emergency Medicine. 2017; 13(1), doi: 10.22514/ sv131.052017.29.

35. Yang CW, Yen ZS, McGowan JE, et al. A systematic review of retention of adult advanced life support knowledge and skills in healthcare providers. Resuscitation. 2012; 83(9): 1055-1060, doi: 10.1016/j. resuscitation.2012.02.027, indexed in Pubmed: 22391016. 